\title{
Fluctuation pressure of biomembranes in planar confinement
}

\author{
Thorsten Auth and Gerhard Gompper \\ Theoretical Soft Matter and Biophysics, Institute of Complex Systems and Institute for Advanced Simulation, \\ Forschungszentrum Jülich, 52425 Jülich, Germany
}

(Received 18 May 2013; published 8 July 2013)

\begin{abstract}
The fluctuation pressure of a lipid-bilayer membrane is important for the stability of lamellar phases and the adhesion of membranes to surfaces. In contrast to many theoretical studies, which predict a decrease of the pressure with the cubed inverse distance between the membranes, Freund suggested very recently a linear inverse distance dependence [Proc. Natl. Acad. Sci. USA 110, 2047 (2013)]. We address this discrepancy by performing Monte Carlo simulations for a membrane model discretized on a square lattice and employ the wall theorem to evaluate the pressure for a single membrane between parallel walls. For distances that are small compared with the lattice constant, the pressure indeed depends on the inverse distance as predicted by Freund. For intermediate distances, the pressure depends on the cubed inverse distance as predicted by Helfrich [Z. Naturforsch. A 33, 305 (1978)]. Here, the crossover length between the two regimes is a molecular length scale. Finally, for distances large compared with the mean squared fluctuations of the membrane, the entire membrane acts as a soft particle and the pressure on the walls again depends linearly on the inverse distance.
\end{abstract}

DOI: 10.1103/PhysRevE.88.010701

PACS number(s): 87.16.A-, 87.16.D-, 82.70.Uv

\section{INTRODUCTION}

The entropic pressure of fluctuating membranes in confinement is the main reason for the finite distance between membranes in a swollen lamellar phase. It plays an important role for membrane adhesion and many other properties of membranes in a confined geometry [1]. The prediction of the fluctuation pressure of membranes by Helfrich [2] with a $D^{-3}$ dependence on the distance $2 D$ between two confining walls has been one of the important early successes for the description of fluid membranes by curvature elasticity. However, in a very recent theoretical and numerical study, Freund [3] concluded that Helfrich's analysis is incorrect, and predicts instead a $D^{-1}$ dependence of the fluctuation pressure. In addition, Sharma [4] has suggested that this prediction should be taken as a stimulus for a new set of experiments.

The prediction of Freund is surprising, because several computer-simulation [5-7], theoretical [8-10], and experimental studies [11,12] seem to have confirmed Helfrich's prediction very well. From an application point of view, lamellar phases of lipid bilayers have been used, for example, to measure the effect of additives, such as proteins or polymers, on the interaction between membranes [13-15]; the interpretation of these results depends strongly on the distance dependence of the fluctuation pressure. Therefore, a profound understanding of lamellar membrane stacks is very important both from a theoretical and an experimental point of view.

\section{MODEL AND METHOD}

Calculations of the fluctuation pressure are based on the curvature elastic energy for nearly planar fluid membranes in the Monge representation,

$$
E_{b}=\frac{\kappa}{2} \int d^{2} r\left[\nabla^{2} h(\mathbf{r})\right]^{2}
$$

where $h(\mathbf{r})$ measures the vertical displacement of the membrane from a planar reference state at horizontal position $\mathbf{r}$.
Here, $\kappa$ is the bending rigidity on the membrane. The walls restrict the height variables within the range $0 \leqslant h(\mathbf{r}) \leqslant 2 D$, so that $2 D$ is the wall separation and $D$ the average distance of the membrane from the wall.

Helfrich predicted the free energy change per unit area due to confinement [2]

$$
\Delta f(D)=c_{1} \frac{\left(k_{B} T\right)^{2}}{\kappa} D^{-2},
$$

which implies a fluctuation pressure

$$
p=-\left[\frac{d \Delta f}{d D}\right]=2 c_{1} \frac{\left(k_{B} T\right)^{2}}{\kappa} D^{-3} .
$$

The universal constant $c_{1}$ was estimated by Helfrich [2] to be $c_{1}=3 / 32=0.094$. Subsequent Monte Carlo simulation studies employed a discretized membrane model, in which continuous height variables $h_{i, j}$ are placed on a $N \times N$ square lattice with lattice constant $a$ with periodic boundary conditions, so that

$$
E_{b}=\frac{\kappa}{2 a^{2}} \sum_{i, j}\left[h_{i+1, j}+h_{i-1, j}+h_{i, j+1}+h_{i, j-1}-4 h_{i, j}\right]^{2},
$$

with the standard discretization of the Laplacian. These simulations gave results consistent with Eq. (2) and yielded the more precise prediction $c_{1}=0.080$ [5,6].

We employ Metropolis Monte Carlo simulations for the model defined by Eq. (4) with $0 \leqslant h(\mathbf{r}) \leqslant 2 D$. From the simulated membrane conformations, we evaluate the density distribution $\rho(z)$ of the membrane between two parallel walls. Here, the density profile is normalized such that

$$
\int_{0}^{2 D} d z \rho(z)=L^{2}
$$

where $L=N a$ is the linear membrane size. A typical membrane conformation obtained from a simulation with $N=100$ is shown in Fig. 1. We calculate the fluctuation pressure directly from the membrane density profile $\rho(z)$, 


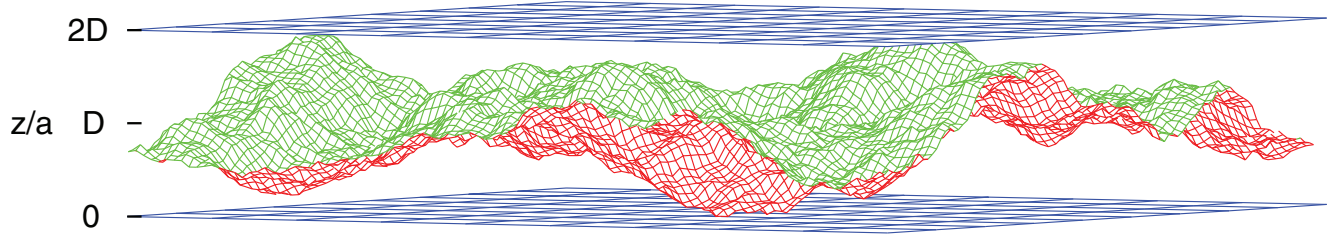

FIG. 1. (Color online) Membrane with $N=100$ fluctuating between two walls at heights $z=0$ and $z=2 D=10 a$. The membrane is drawn as a mesh that is colored green (light gray) on its upper and red (dark gray) on its lower side.

which are related by the wall theorem $[16,17]$,

$$
p=k_{B} T \rho_{w},
$$

where $\rho_{w}$ is the density at the wall.

\section{RESULTS}

In Fig. 2, the membrane density profile $\rho(z)$ is shown for a "small," an "intermediate," and a "large" distance of the parallel walls. The boundaries between these three regimes will be discussed and quantified below. For small distances, the density varies only very slightly as function of the vertical position $z$ between the walls. For intermediate distances, the density profile is described very well by a Gaussian function [18,19] (see Fig. 2). The membrane that fluctuates between hard walls can be approximated by a membrane that fluctuates in a parabolic potential [18], where an additional term $V(h)=(K / 2) h^{2}$ is added to Eq. (1). The potential strength for the parabolic potential has been chosen to be $K=\left(k_{B} T\right)^{2} /\left(\kappa b^{4} D^{4}\right)$, where $b \approx 1.14$. Thus $\left\langle h^{2}\right\rangle \approx 0.16 D^{2}$, which is consistent with Ref. [19]. For large distances, the density profile is flat in the bulk and decreases towards the walls, as expected for a soft particle. The size of the effective soft particle is given by the typical mean squared fluctuations of the membrane, $\left\langle h^{2}\right\rangle^{1 / 2}=\left(4 \pi^{3 / 2}\right)^{-1} L\left(k_{B} T / \kappa\right)^{1 / 2}$, of the membrane of linear size $L$.

The resulting dependence of the fluctuation pressure on the wall distance is shown in Figs. 3-5 for various system sizes $N$,

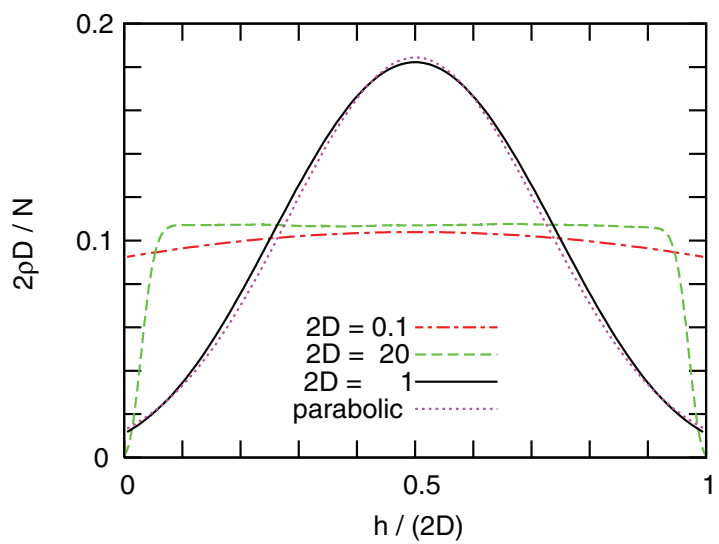

FIG. 2. (Color online) Membrane density profile $\rho(z)$ between two walls at $z=0$ and $z=2 D$. The bending rigidity is $\kappa / k_{B} T=5$. The density is normalized by the number of lattice sites where the membrane is defined and the separation of the walls is given in the legend. The density distribution for $2 D / a=1$ is compared with that of a membrane that fluctuates in a parabolic potential. discretization lengths $a$, and bending rigidities $\kappa$. Analogously to the density distributions in Fig. 2, we find three power-law regimes: a small- $D$ regime with $p \sim D^{-1}$, an intermediate$D$ regime with $p \sim D^{-3}$, and a large- $D$ regime with $p \sim$ $D^{-1}$. The fluctuation pressure for small wall distances does not depend on the membrane size $N$ and on the bending rigidity $\kappa$. It is given by

$$
p=k_{B} T /\left(2 D a^{2}\right),
$$

which is the ideal-gas pressure of $N^{2}$ independently fluctuating height variables, in agreement with Ref. [3]. In the regime of intermediate wall distances, the fluctuation pressure follows the Helfrich prediction (3), as can best be seen in Fig. 3, where the pressure dependence is shown for different system sizes $N$. A fit of Eq. (3) gives the universal amplitude $c_{1}=$ $0.081 \pm 0.002$, in agreement with previous simulation results $[5,6]$. Note that in this regime the pressure is independent of the discretization length $a$. For large distances, the pressure is dominated by the translational degree of freedom of the entire membrane, so that

$$
p=\alpha k_{B} T /\left(2 D L^{2}\right)
$$

with a function $\alpha(\kappa)=1+O\left(\sqrt{k_{B} T / \kappa}\right)$. The parameter $\alpha$ depends only weakly on the bending rigidity (see Fig. 5). For further analysis we use $\alpha=1$.

We can look at the simulation results from three different perspectives. For fixed $\kappa$ and $a$, the finite-size regime shifts to larger $D$ for increased $L$. The finite-size regime is reached when the lateral correlation length $\xi_{\|}$reaches the system

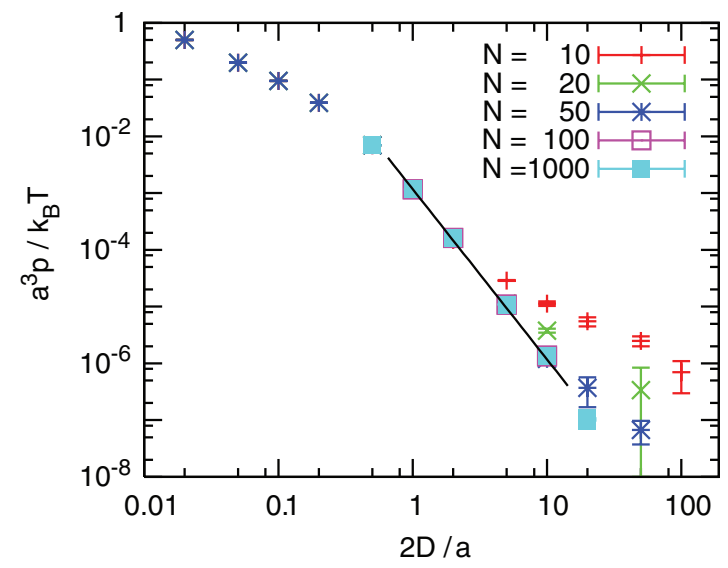

FIG. 3. (Color online) Membrane pressure $p$ as function of the distance $2 D$ between the walls for fixed $a, \kappa=5 k_{B} T$, and several membrane sizes $N$. The line is a fit to Eq. (3) with the fit parameter $c_{1}$. 


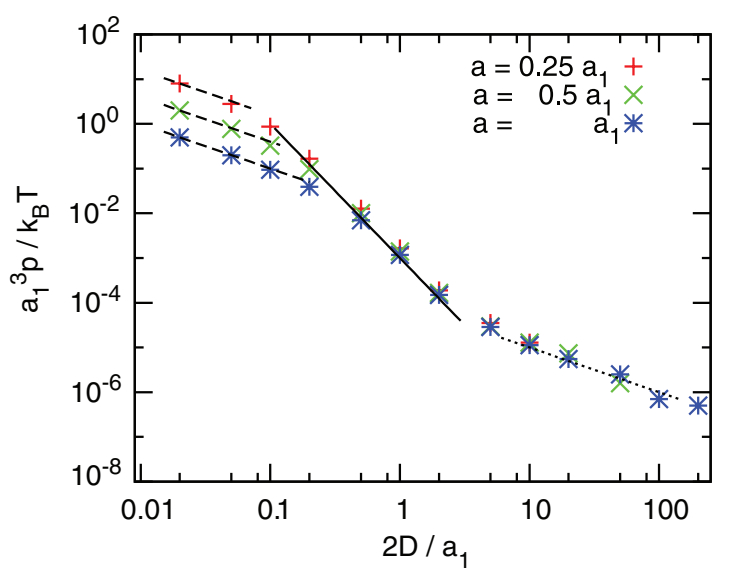

FIG. 4. (Color online) Membrane pressure $p$ as function of the distance $2 D$ between the walls. Two systems with a smaller discretization length are compared with a reference system with $L_{1}=10 a_{1}$ for fixed $L$ and $\kappa=5 k_{B} T$. The lines indicate the power laws of Eqs. (7), (3), and (8), by dashed, solid, and dotted lines, respectively.

size $L$. Here, $\xi_{\|}$is defined by decay of the height-height correlation function $\left\langle h(\mathbf{r}) h\left(\mathbf{r}^{\prime}\right)\right\rangle \sim \exp \left(-\left|\mathbf{r}-\mathbf{r}^{\prime}\right| / \xi_{\|}\right)$, and can be interpreted as the average size of the largest membrane humps (see Fig. 1). It has been shown that $\xi_{\|} \sim\left(\kappa / k_{B} T\right)^{1 / 2} D$ $[1,18]$. The crossover system size can also be obtained by equating Eqs. (3) and (8), which gives the more precise estimate

$$
D_{\mathrm{fs}}=\left(2 c_{1} / \alpha\right)^{1 / 2} L\left(k_{B} T / \kappa\right)^{1 / 2},
$$

with prefactor $\left(2 c_{1} / \alpha\right)^{1 / 2} \approx 0.4$. Alternatively, we can consider a system of fixed lateral size $L=N a$ and fixed bending rigidity $\kappa$, and vary the discretization length $a$ (and accordingly the number of $N^{2}$ height variables). This implies that the prefactor $\kappa / a^{2}$ in the discretized curvature energy in Eq. (4) also varies. The results are shown in Fig. 4. In this case, the finite-size and the Helfrich regime are unaffected by decreasing $a$, but the small- $D$ regime moves to smaller and smaller values of $D$. This implies that the small- $D$ regime

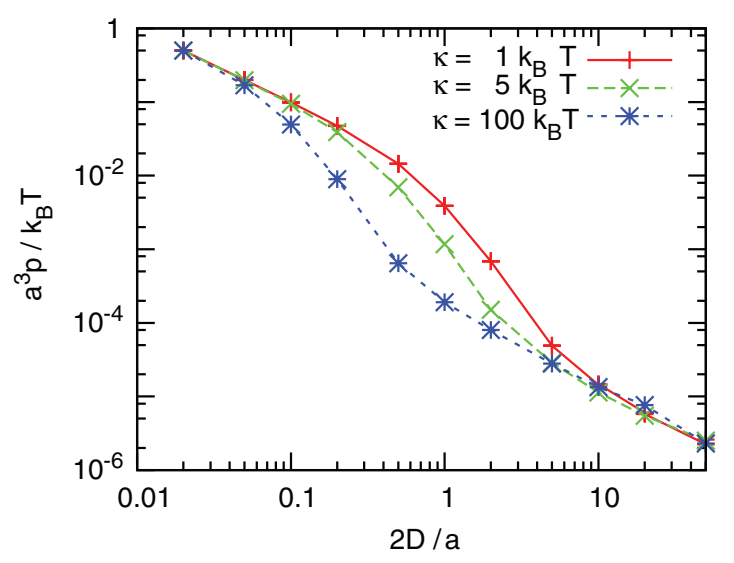

FIG. 5. (Color online) Membrane pressure $p$ as function of the distance $2 D$ between the walls for a membrane with $N=10$, fixed $a$, and various bending rigidities $\kappa$. Lines are guides to the eye. vanishes in the continuum limit. The regime of independently fluctuating height variables is entered with decreasing $D$ when the lateral correlation length $\xi_{\|} \sim\left(\kappa / k_{B} T\right)^{1 / 2} D$ of the continuum model drops below the lattice constant $a$, so that $D_{\mathrm{mol}} \sim\left(k_{B} T / \kappa\right)^{1 / 2} a$. A more precise estimate can again be obtained by equating Eqs. (3) and (7), which yields

$$
D_{\mathrm{mol}}=\left(2 c_{1}\right)^{1 / 2} a\left(k_{B} T / \kappa\right)^{1 / 2},
$$

with prefactor $\left(2 c_{1}\right)^{1 / 2} \approx 0.4$. This result also shows that the discretization crossover shifts to smaller values of $D$ with increasing $\kappa$, as shown explicitly in Fig. 5.

\section{SUMMARY AND CONCLUSIONS}

We have performed large-scale Monte Carlo simulations with up to 1000000 lattice sites to clarify the discrepancy between the predictions of Freund [3] and Helfrich [2] for the fluctuation pressure of membranes. We employ a discretized version of the membrane curvature energy with short-distance cutoff $a$. This length scale is not an artifact of the simulation approach, but can be seen as a way to mimic the breakdown of the continuum description on a molecular level, where the motion of individual lipid molecules becomes important [20,21]. These molecular motions have often been denoted as "protrusion modes." We want to emphasize that the discretized curvature model is not a very good model for the protrusion regime, but should still capture the crossover between the two regimes.

The main result of our simulations is that although the fluctuation pressure of a membrane indeed follows a $D^{-1}$ behavior for very small $D$, the curvature elasticity of the membrane plays no role in this regime and the pressure is generated by independent motion of individual "molecules." The small- $D$ regime occurs when the height differences of neighboring lattice sites are too small to be controlled by the bending rigidity. The wall distance $2 D_{\text {mol }}$, below which the pressure shows a $D^{-1}$ behavior, is always smaller than the cutoff length $a$ (for $\kappa / k_{B} T \geqslant 1$ ) and decreases with increasing $\kappa$. For wall distances larger than the molecular length scale, we confirm the $D^{-3}$ distance dependance of the pressure predicted by Helfrich, with the universal amplitude $c_{1}=0.080$. This curvature-elasticity controlled behavior is seen in the regime $a<\xi_{\|}<L$.

Interestingly, when the data in Fig. 4 of Ref. [3] is replotted in a double-logarithmic presentation, a crossover becomes visible from $p \sim D^{-1}$ to a faster decay. The value of the crossover distance should depend on the number of fluctuation modes employed in the analysis. We find that the length scale $\lambda$ in the model proposed by Freund corresponds to our molecular cutoff length $a$. In contrast, Sharma [4] claims that $\lambda$ is large compared with the molecular size and therefore the use of the continuum model is justified.

In conclusion, the analysis of previous experiments on the basis of the theoretical expression for the fluctuation pressure with an inverse cubic dependence on the confinement remains valid-for wall distances corresponding to parallel correlation lengths in the range $a<\xi_{\|}<L$. For smaller $D$ (with $\xi_{\|}<a$ ), we confirm an inverse linear dependence of the fluctuation 
pressure. Therefore, it would be very interesting to perform new experiments and molecular simulations to investigate the crossover from the undulation- to the protrusion-dominated regime.

\section{ACKNOWLEDGMENT}

Stimulating discussions with S. Dietrich (Stuttgart) are gratefully acknowledged.
[1] Handbook of Biological Physics, edited by R. Lipowsky and E. Sackmann (Elsevier, Amsterdam, 1995), Vol. 1.

[2] W. Helfrich, Z. Naturforsch. A 33, 305 (1978).

[3] L. B. Freund, Proc. Natl. Acad. Sci. USA 110, 2047 (2013).

[4] P. Sharma, Proc. Natl. Acad. Sci. USA 110, 1976 (2013).

[5] W. Janke, H. Kleinert, and M. Meinhart, Phys. Lett. B 217, 525 (1989).

[6] G. Gompper and D. Kroll, Europhys. Lett. 9, 59 (1989).

[7] R. R. Netz and R. Lipowsky, Europhys. Lett. 29, 345 (1995).

[8] H. Kleinert, Phys. Lett. A 257, 269 (1999).

[9] M. Bachmann, H. Kleinert, and A. Pelster, Phys. Lett. A 261, 127 (1999).

[10] B. Kastening, Phys. Rev. E 73, 011101 (2006).

[11] C. R. Safinya, D. Roux, G. S. Smith, S. K. Sinha, P. Dimon, N. A. Clark, and A. M. Bellocq, Phys. Rev. Lett. 57, 2718 (1986).
[12] D. Roux and C. Safinya, J. Phys. (France) 49, 307 (1988).

[13] G. Bouglet and C. Ligoure, Eur. Phys. J. B 9, 137 (1999).

[14] J. Brooks and M. Cates, J. Chem. Phys. 99, 5467 (1993).

[15] A. Giahi, M. E. A. Faris, P. Bassereau, and T. Salditt, Eur. Phys. J. E 23, 431 (2007).

[16] J. P. Hansen and I. R. McDonald, Theory of Simple Liquids, 3rd ed. (Academic, London, 2006).

[17] G. Gompper and D. M. Kroll, J. Phys. I (France) 1, 1411 (1991).

[18] S. Leibler and R. Lipowsky, Phys. Rev. B 35, 7004 (1987).

[19] G. Gompper and D. M. Kroll, Europhys. Lett. 15, 783 (1991).

[20] R. Goetz, G. Gompper, and R. Lipowsky, Phys. Rev. Lett. 82, 221 (1999).

[21] E. Lindahl and O. Edholm, Biophys. J. 79, 426 (2000). 